\title{
Acute Radiation-Induced Changes in Sprague-Dawley Rat Submandibular Glands: A Histomorphometric Analysis
}

\author{
Manu Krishnan ${ }^{\mathrm{a}, \mathrm{f}}$, Aatish Tennavan ${ }^{\mathrm{a}}$, Seema Saraswathy ${ }^{\mathrm{b}}$, Tarun Sekhric, \\ Ajay Kumar Singh ${ }^{\mathrm{d}}$, Velu Nair
}

\begin{abstract}
Background: Radiation-induced xerostomia is a distressing clinical condition that starts appearing from the initial stages of radiotherapy in head and neck cancer patients. Though submandibular glands contribute to maximum of the "resting salivary" secretions, most of the acute xerostomia experiments so far reported have been on animal parotid glands. Therefore, we assessed and quantified the histologic changes in submandibular glands of Sprague-Dawley (SD) rats using histomorphometry, 24 hours after radiation.
\end{abstract}

Methods: Three SD rats were given single-dose radiation of 15 Gray from a gamma cobalt-60 irradiator. Same number of non-radiated animals was the controls. Animals were sacrificed at 24 hours followed by histopathology and histomorphometry of submandibular glands, where the mean values were analyzed by Student's $t$-test.

Results: Irradiated submandibular glands showed highly significant reduction in acinar area (53\%: $77.16 \pm 5.05 \%$ to $36.55 \pm 4.90 \%)$ and acinar size ( $87 \%$ : $3,447.53 \pm 461.03 \mathrm{~mm}^{2}$ to $\left.428.25 \pm 75.22 \mathrm{~mm}^{2}\right)$ with concomitant increase in inter-acinar space $(3.46 \pm 0.67 \mathrm{~mm}$ to 10.08 $\pm 0.60 \mathrm{~mm}$ ). Acini nuclei displayed anisonucleosis, poikilonucleosis and pyknosis. "Serous acini" had marked morphologic changes, with fluid accumulation between cells, generalized cytoplasmic vacuolation and vascular congestion, while "mucous acini" largely retained

Manuscript accepted for publication March 30, 2017

aDepartment of Dental Research \& Implantology, Institute of Nuclear Medicine and Allied Sciences (INMAS), Defence Research and Development Organization (DRDO), Delhi, India

${ }^{\mathrm{b} S}$ School of Medicine and Paramedical Health Sciences, Guru Gobind Singh Indraprastha University, Delhi, India

'Division of Health, Endocrinology, Thyroid and Dental Research, Institute of Nuclear Medicine and Allied Sciences (INMAS), Defence Research and Development Organization (DRDO), India

${ }^{\mathrm{d}}$ Radiation Biosciences Division, Institute of Nuclear Medicine and Allied Sciences (INMAS), Defence Research and Development Organization (DRDO), Delhi, India

${ }^{\mathrm{e}}$ Army Hospital Research \& Referral (AHRR), Delhi, India

${ }^{\mathrm{f} C o r r e s p o n d i n g ~ A u t h o r: ~ M a n u ~ K r i s h n a n, ~ D e p a r t m e n t ~ o f ~ D e n t a l ~ R e s e a r c h ~ \& ~}$ Implantology, Institute of Nuclear Medicine and Allied Sciences (INMAS), Defence Research and Development Organization (DRDO), Delhi, India. Email: manuseema13@gmail.com

doi: https://doi.org/10.14740/wjon1021w cell architecture. Similarly, ductal cells and nuclei also did not show apparent differences. This demonstrated radiosensitivity variations among different submandibular gland cell types.

Conclusion: Evaluation of acute submandibular acinar cell dysfunctions has helped in quantifying the histologic elements, which mainly contribute to the resting salivary secretions. Findings would aid in future research of radioprotector drugs, salivary glandular regeneration modalities and in devising prudent radiotherapy protocols to address radiation-induced xerostomia.

Keywords: Radiation-induced xerostomia; Submandibular glands; Sprague-Dawley rats; Histomorphometry

\section{Introduction}

Intensity modulated radiotherapy (IMRT) in combination with surgery and chemotherapy forms the contemporary standard in treating head and neck cancers. Ionizing radiations in fractions of 2 - 3 Gray (Gy) per day, in a cycle of 5 days per week, totaling to $30-80 \mathrm{~Gy}$, are used for the destruction of cancer cells, aimed with little damage to the normal cells [1]. However, the major (paired) parotid, submandibular, sublingual and minor (numerous and widely distributed over the oral mucosa) salivary glands are inadvertently exposed in head and neck cancer radiotherapy. Though in current IMRT protocols, parotids are spared, submandibular glands are generally overlooked [2]. Radiation-induced side effects of salivary glands are acute and chronic. Acute effects occur during radiotherapy while chronic complications arise at the completion of it. Acute problems are xerostomia, oropharyngeal mucositis, speech disturbances, heightened risk for oral infections and dental caries, periodontal pain, difficulties in swallowing (dysphagia) and altered taste sensation (dysguesia). Late effects include xerostomia, trismus, fibrosis and osteoradionecrosis [3]. Xerostomia or diminished salivary secretion is a debilitating oral condition that persists both in the acute and chronic phase. It seriously compromises nutrition, general health and thereby the quality of life (QoL) of the patient. Additionally, it disrupts the radiotherapy schedule [4]. The current understanding on xerostomia has come from salivary gland histologic studies of rats, mice, rhesus monkeys and mini-pigs done after controlled irradiation 
[3]. But most of the investigations have been with parotids and few with submandibular glands.

Salivary glands have two components: myoepithelial cells with its "serous" and "mucous" acini, invested by the ductal system. The fluid and protein part of saliva are expressed by the acinar cells and the inorganic part by the salivary duct. Secretory end pieces from the acini drain into the intercalated ducts, which sequentially increase in diameter to the striated and finally the excretory ducts and modify the salivary composition by selective absorption and secretion [5]. Saliva is mainly composed of water $(99.5 \%)$, proteins, electrolytes and antimicrobials. It has important functions: epithelial hydration, facilitate mastication, taste, swallowing and speech. It has a buffering action with the bicarbonate ions that prevents enamel decalcification while promoting remineralization. A total of $1.5 \mathrm{~L}$ of saliva is produced by the major salivary glands: parotid: $20-25 \%$, submandibular: $70-75 \%$, sublingual: $5 \%$ and the rest by the minor salivary glands. Most of the resting or un-stimulated saliva is from the submandibular glands, while parotids contribute only to the stimulated secretions [6]. After radiation, sodium and chloride concentrations increase, while bicarbonates decrease. This lowers the $\mathrm{pH}$ and affects the buffering capacity of saliva, making tooth susceptible for caries [4]. Salivary acinar cells are predominantly in post-mitotic phase and therefore are not so radiosensitive. But in terms of the drastic reduction in salivary volume and alterations in its composition in the acute phase, they are radiosensitive also. So in the acute phase, salivary glands exhibit a dual behavior; with respect to salivary changes, it mimics to radiation response shown by "higher mitotic index" bone marrow and intestine, but from the basis of cell death, it is akin to "slow responding tissues" like lungs $[3,7]$. Stem cells and progenitor cells are another cell component in the salivary glands. Stem cells reside amongst excretory and striated ducts, while progenitor cells are between the striated and intercalated ducts. The stem cell pool generates the progenitor cell types, which in turn replaces the acinar and ductal cells. Radiation damages the stem and progenitor cells also [6].

The current radiotherapy regimes for head and neck cancers are kept at $2 \mathrm{~Gy} /$ day to keep a balance between the most favorable therapeutic effects and in ensuring recovery of salivary functions. With a 5-day weekly schedule, this works out to a cumulative dose of $10 \mathrm{~Gy} /$ week [7]. However, there is wide disparity in the animal experiment models regarding radiation doses and time points. Different single dose ranges used for animal studies are: rat: 5 - $40 \mathrm{~Gy}$, mouse: 1 - $15 \mathrm{~Gy}$, rhesus monkey: 2.5 - 15 Gy and mini pig: 15 - 20 Gy [3]. It follows that there is lack of consensus on the right animal vs. human dose for experimental evaluation of radiotherapy. Levels of salivary gland dysfunction against the number and area of acinar cells affected in the acute phase are also not known. This is important to investigate because acute effects initiate inflammatory reactions and oxidative stress in the acinar microenvironment, which subsequently manifest as late effects [8].

Since submandibular gland contributes to majority of the resting saliva secretion, maintaining submandibular gland functions would be central towards reversing radiation effects sustained during head and neck cancer radiotherapy and in improving the QoL of patients. However, they have been less histologically studied after radiation, compared to parotids. Immediate post-radiation exposure studies would help in identifying the key histologic features, which can be given due recognition in the emerging IMRT protocols. It would also give right directions in formulating suitable radioprotector drugs for mitigating the radiation effects in the crucial first $24 \mathrm{~h}$. Surgical attempts to relocate submandibular salivary glands from the path of radiotherapy are also a testimony to the importance of protecting this gland from unintended radiation [9]. Rat submandibular histologic studies at $24 \mathrm{~h}$ would be a prelude for all the above. Therefore, aim of the present study was to evaluate the histologic changes in submandibular glands of Sprague-Dawley (SD) rats using histomorphometry at $24 \mathrm{~h}$ time point, following single dose of 15 Gy irradiation, to provide reproducible baseline data for future biological and therapeutic studies in radiation-induced xerostomia.

\section{Materials and Methods}

\section{Animal irradiation experiments}

They were designed as per European Communities Council Directives (86/609/EEC) and were performed after receiving the institutional animal ethics committee approval. Three, 4 - 5 months old SD rats weighing 300 - $350 \mathrm{~g}$ were used for the irradiation. Same number of non irradiated rats served as the controls. In rats, the submandibular gland is located alongside the sublingual gland in the anterior neck area superior to the submandibular lymph nodes and the masseter muscle and inferiorly to the sternum. Midline of the head and neck of the animal, corresponding to this gross surgical anatomy including both the right and left side glands was selected for the irradiation field. For focused radiation, animals were anesthetized with ketamine $(80 \mathrm{mg} / \mathrm{kg}$ body weight) and xylazine $(10 \mathrm{mg} /$ $\mathrm{kg}$ body weight) and secured in supine position on a perspex sheet to minimize pain and discomfort, under the collimated gamma cobalt-60 irradiator (Bhabhatron-2, Panasia Medical Technology India).

Rats were given a non-fractionated, single dose of $15 \mathrm{~Gy}$ at the rate of $0.83 \mathrm{~Gy} / \mathrm{min}$. Irradiation procedure took around 18 min for each animal. After irradiation, animals were transferred to an autoclaved cage and observed regularly at $1 \mathrm{~h}$ intervals. During this period, they were fed on soft diet. After $24 \mathrm{~h}$, animals were sacrificed in a carbon dioxide pressurized chamber. Dissection of the salivary glands was done immediately and the glands were kept in 10\% neutral buffered formalin for $24 \mathrm{~h}$ for adequate fixation.

\section{Histopathology}

Tissues were dehydrated in increasing grades of ethyl alcohol $(70 \%, 95 \%$, and $100 \%)$, followed by treatment with xylene. They were then embedded in paraffin. Tissue sections were cut at $4 \mu \mathrm{m}$ thickness, stained with hematoxylin and eosin (H\&E), mounted using DPX mounting medium (Sigma Aldrich, St. 

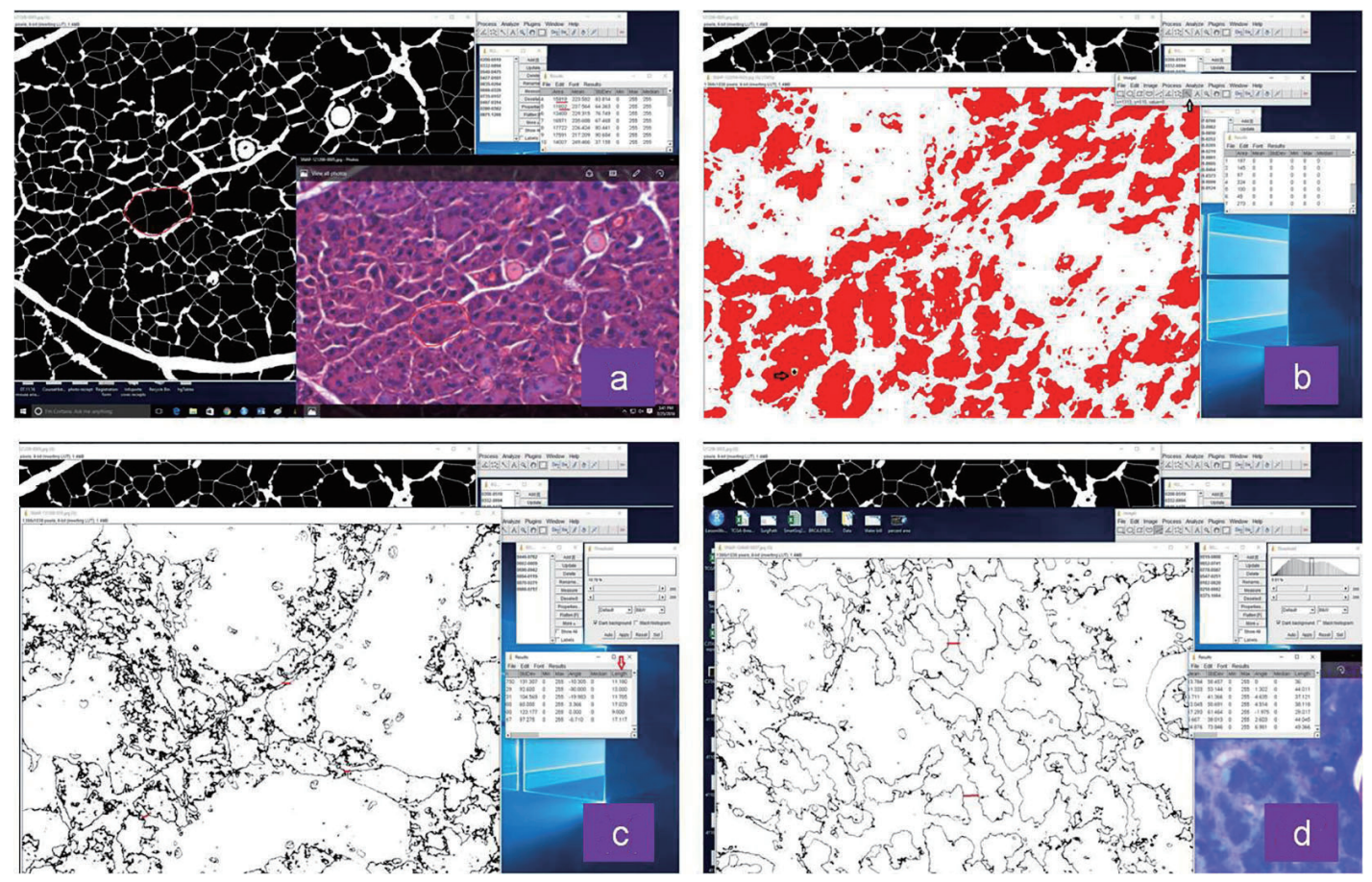

Figure 1. Representative images of histomorphometric analysis of normal and irradiated glands by Image $\mathrm{J}$ software: (a) acinar size, (b) vacuole size, (c) distance between acini in non-irradiated and (d) irradiated group.

Louis, MO, USA) and viewed under light microscope (Carl Zeiss, Jena, Germany).

\section{Histomorphometry}

This was done by examining random areas in the slides for the following: acinar area and size, presence/absence/size of vacuoles, distance between each acinus, ductal area, acinar and ductal nuclear size, ductal dilation, blood vessel congestion, neural tissues, fat cells and adiposis. Nuclear changes in the acini and the ducts: size/shape of nucleus, type of chromatin, presence of nucleoli and nuclear pyknosis were also evaluated. All analyses were done on photomicrographs using Image $\mathrm{J}$ software, version 16 (National Institutes of Health, Bethesda, MD, USA). Measurements were initially done with black and white images. Subsequently, H\&E images were converted by splitting the channels to the requisite color and the thresholds were adjusted specifically for acinar and ductal area. The acinar size was determined as the two-dimensional area of individual serous acinus by applying "watershed partitioning boundaries". For gauging two-dimensional area of vacuole "wand tool" was preferred since the vacuoles were of circular to oval in shape. The dimensions were converted from pixels to millimeter by the software.

\section{Statistical analysis}

Data were analyzed using Statistical Package for Social Sciences (SPSS) version 20.0 by IBM Corp. Values were repre- sented as mean \pm standard deviation and were compared by Students' $t$-test. For all statistical evaluation, a two-tailed probability of value $\mathrm{P}<0.05$ was considered significant.

\section{Results}

Representative images of histomorphometric analysis of normal and irradiated glands by Image $\mathrm{J}$ software are shown in Figure 1. Under light microscopy, H\&E sections of control submandibular glands showed characteristic secretory acinar histology. They were mainly of the serous type and were composed of pyramidal cells surrounding a narrow lumen with foamy basophilic cytoplasm and a rounded basal nucleus. Duct system consisted of intercalated ducts and granular convoluted tubules (GCTs) with columnar cells containing excretory granules and ducts. Normal neurovascular bundle was seen close to the excretory ducts. Irradiated submandibular glands exhibited acinar atrophy and shrinkage with varying degrees of ductal (GCT) degeneration (Fig. 2). Secretory acinar cells also showed generalized cytoplasmic vacuolation and increased acinar widening, though mucous acinar cells retained their shape and morphology (Fig. 3). Details of morphometric analysis are shown in Table 1.

Serous acinar area was calculated as overall percentage of acinar cell compartment in the H\&E photomicrograph. Acinar area was reduced from $77 \%$ to $36 \%$ with shape change: rounded to angulated and shrunken morphology. The mean serous acinar cell size displayed a significant $(\mathrm{P}<0.001)$ shrinkage from $3,447.53 \pm 461.03$ to $428.25 \pm 75.22 \mathrm{~mm}^{2}$. Numerous vacuoles were seen which occupied an average area of $56.18 \pm$ 


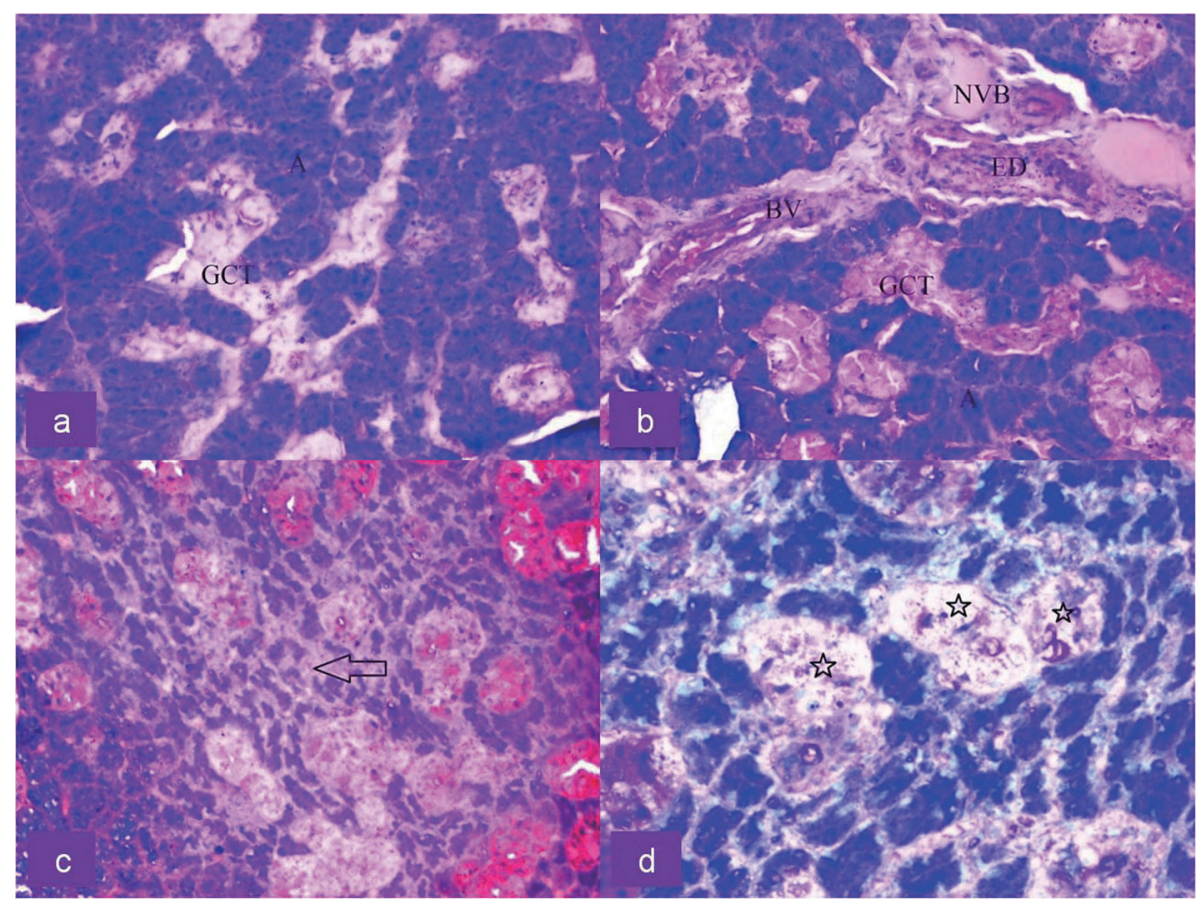

Figure 2. Upper row: H\&E-stained section of submandibular gland of control rat showing (a) dark serous acini (A) and light stained granular convoluted tubule (GCT) duct cells and (b) showing neurovascular bundle (NVB), blood vessels (BV), excretory duct (ED) and GCT at $\times 20$ magnification. Lower row: H\&E-stained section of irradiated submandibular gland showing (c) shrunken serous acini (arrow) with increased inter-acinar space at $\times 20$ magnification and (d) degeneration of GCT duct cells (star) at $\times 40$ magnification.

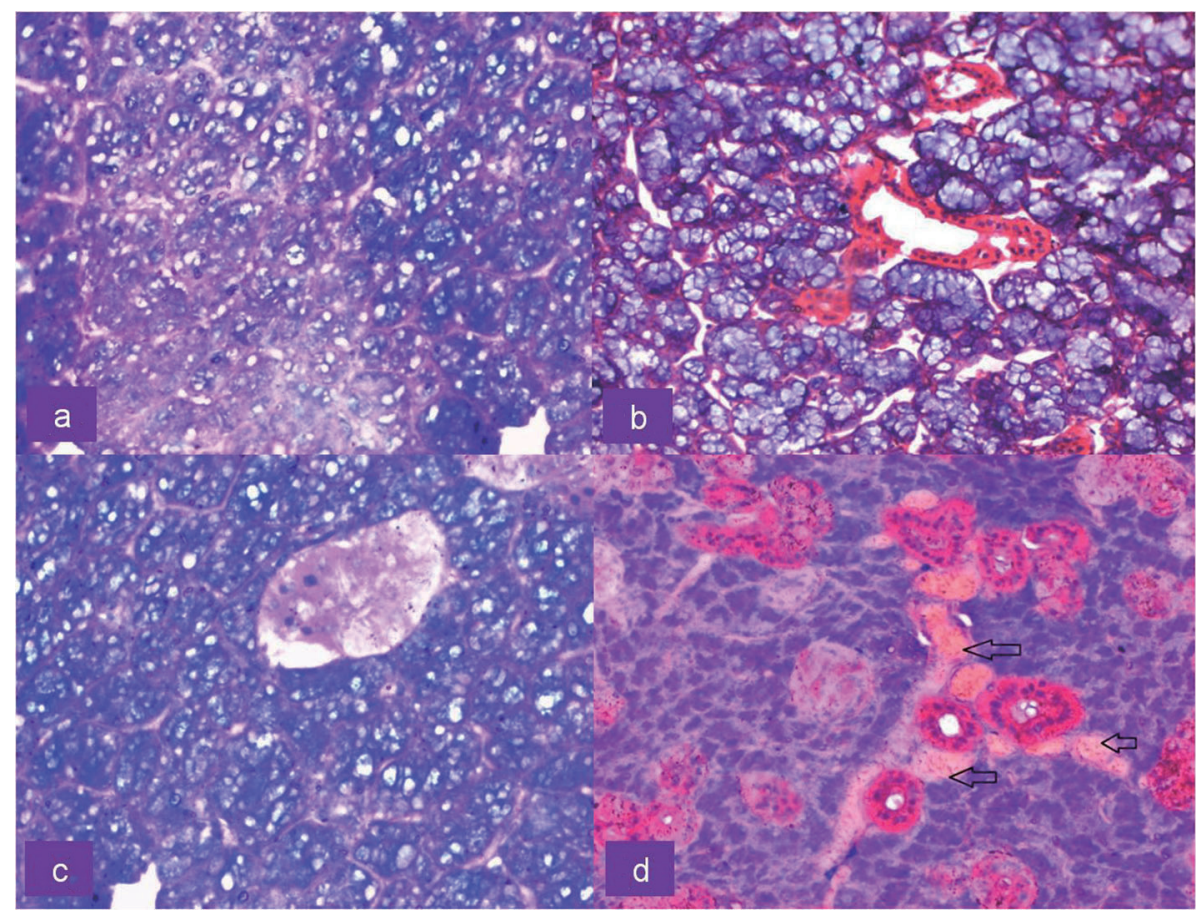

Figure 3. Upper row: H\&E-stained section of irradiated submandibular gland showing (a) cytoplasmic vacuolation of serous acini at $\times 40$ magnification and (b) mucous acini exhibiting no morphological alterations at $\times 20$ magnification. Lower row: H\&E-stained section of irradiated serous acini showing (c) pyknotic nuclei and nuclear fragmentation along with vacuolation at $\times 40$ magnification and (d) congestion of blood vessels (arrows) at $\times 20$ magnification. 
Table 1. Morphometric Analysis

\begin{tabular}{|c|c|c|}
\hline Feature & Control & Irradiated \\
\hline Serous acinar area $(\%)^{*}$ & $77.16 \pm 5.05$ & $36.55 \pm 4.90$ \\
\hline Serous acinar size $\left(\mathrm{mm}^{2}\right)^{*}$ & $3,447.53 \pm 461.03$ & $428.25 \pm 75.22$ \\
\hline Serous acinar shape & Round & Angulated \\
\hline Vacuoles & Absent & Present \\
\hline Vacuole features* & - & Round to ovoid in shape; $56.18 \pm 18.62 \mathrm{~mm}^{2}$ in area \\
\hline Space between serous acini $(\mathrm{mm})^{*}$ & $3.46 \pm 0.67$ & $10.08 \pm 0.60$ \\
\hline Acinar degeneration & Absent & Acinar shrinkage \\
\hline Ductal area (percentage) & - & Nuclear changes seen without much architectural change \\
\hline Ductal dilation & Absent & Absent \\
\hline Ductal degeneration & Absent & Present \\
\hline Acinar nuclear size $\left(\mathrm{mm}^{2}\right)^{*}$ & $137.44 \pm 25.66$ & Nuclear pyknosis \\
\hline Ductal nuclear size $\left(\mathrm{mm}^{2}\right)^{*}$ & $50.15 \pm 29.23$ & $53.63 \pm 21.14$ \\
\hline Type of chromatin & Euchromatin & Euchromatin to heterochromatin \\
\hline Shape of acinar nucleus & Round to ovoid & Flattened-shrunken \\
\hline Shape of ductal nucleus & Round to ovoid & Round to ovoid \\
\hline Blood vessel morphology & Normal & Normal \\
\hline Blood vessel congestion & Absent & Present \\
\hline Neural tissue & Normal & Normal \\
\hline Fat cells & Not seen & Not seen \\
\hline Collagen fiber sclerosis & Not seen & Not seen \\
\hline Mucous acini & Normal & Normal \\
\hline
\end{tabular}

${ }^{*}$ The values given are as mean \pm SD.

$18.62 \mathrm{~mm}^{2}$ in randomly selected areas of each sample. There was a significant increase in the inter-acinar space compared to the controls: $10.08 \pm 0.60$ vs. $3.46 \pm 0.67 \mathrm{~mm}$. Ductal cells showed degeneration at places with few nuclear changes. Fragmentation was present. Endothelial cells and neural tissue did not exhibit much change except for the congestion of blood vessels (Fig. 3). Nuclei of irradiated gland acini exhibited varied size (anisonucleosis) and shape (poikilonucleosis) with irreversible condensation of the chromatin (pyknotic) nuclei. However, nuclei of the irradiated gland ducts were not affected. No significant difference was noticed in the ductal nuclear size. No adipose tissue was noticed in any of the irradiated samples. The comparison of histomorphometric parameters is given in Table 2. Box plots describing the mean values and the interquartile range are shown in Figure 4.

\section{Discussion}

Radiation effects within the first 30 days are called as acute radiation syndrome (ARS). It has a similar pattern for humans and rodents with its two components: hematopoietic syndrome occurs in response to 3 - 8 Gy characterized by thrombocytopenia and neutropenia, while gastrointestinal syndrome develops at 8 - 20 Gy along with fluid/electrolyte imbalance and sepsis [10]. Radiation dose of whole body exposure required to re- duce survival to $50 \%$ by day 30 without any medical assistance is known as lethal dose (LD) 50/30 values, which is 3.5 - 4 Gy for humans against 7 - 9 Gy for rodents [11]. Also, for humans, the maximum cumulative dose permissible for submandibulars (39 Gy) is higher compared to parotids (24 - $26 \mathrm{~Gy}$ ) [12]. With this logic, we decided on a single dose of 15 Gy for the animal study, half of which would be equal to the radiation delivered to humans in 2 - 3 days ( 2 Gy/day in a 5-day weekly schedule), from which reliable estimates of submandibular gland effects could be derived.

We found that within $24 \mathrm{~h}$ itself, rat submandibular glands showed distinct histologic changes. The serous acinar size and area showed marked changes after irradiation. Acini also showed morphologic alterations with increased spacing between the cells. Mofty and Kahn were the first to report the 24 $\mathrm{h}$ radiation effects in rat parotids in the early 1980s: glandular necrosis at 2 - 20 Gy [13]. However, we did not see any necrosis in the submandibular gland sections with 15 Gy. This demonstrated the radio resistance of submandibular glands compared to parotids. With $15 \mathrm{~Gy}$, a dose that simulated to an actual clinical scenario of 3 - 4 days of fractionated radiotherapy, we observed intact "mucous" cell architecture against "serous" acinar atrophy. This brought out the differences in radiosensitivity among submandibular cell types. However, at 96 h, after giving 50 Gy, Abok and co-workers found irreversible damages in the rat submandibular serous cells [14]. We 
Table 2. Comparison of Histomorphometric Parameters Between Control and Irradiated Groups

\begin{tabular}{llll}
\hline Parameter & Group & Mean \pm SD & t value \\
\hline Serous acinar area $(\%)$ & Control & $77.16 \pm 5.05$ & $16.326^{* *}$ \\
Serous acinar size $\left(\mathrm{mm}^{2}\right)$ & Irradiated & $36.55 \pm 4.90$ & \\
& Control & $3,447.53 \pm 461.03$ & $18.281^{* *}$ \\
Vacuole features $\left(\mathrm{mm}^{2}\right)$ & Irradiated & $428.25 \pm 75.22$ & - \\
\hline Space between serous acini $(\mathrm{mm})$ & Control & - & \\
& Irradiated & $56.18 \pm 18.62$ & $-20.821^{* *}$ \\
Acinar nuclear size $\left(\mathrm{mm}^{2}\right)$ & Control & $3.46 \pm 0.67$ & - \\
\hline Ductal nuclear size $\left(\mathrm{mm}^{2}\right)$ & Irradiated & $10.08 \pm 0.60$ & \\
& Control & $137.44 \pm 25.66$ & -0.272 \\
\hline
\end{tabular}

${ }^{* *} \mathrm{P}<0.001$

presumed that radiation parameters of their study too high for deciphering early changes. Previous studies focusing on early changes (within 1 week) had consensus with us on: serous acinar destruction, ductal degranulation and cytoplasmic vacuolization $[15,16]$. However, using morphometry, we could spe- cifically determine the reduction in acinar size (87\%) and area $(53 \%)$ of salivary gland parenchyma, $24 \mathrm{~h}$ after irradiation.

The sparing of mucous acini compared to serous acini is attributed to the increased radiosensitivity of latter due to the presence of heavy metals like zinc, iron and copper ions con-

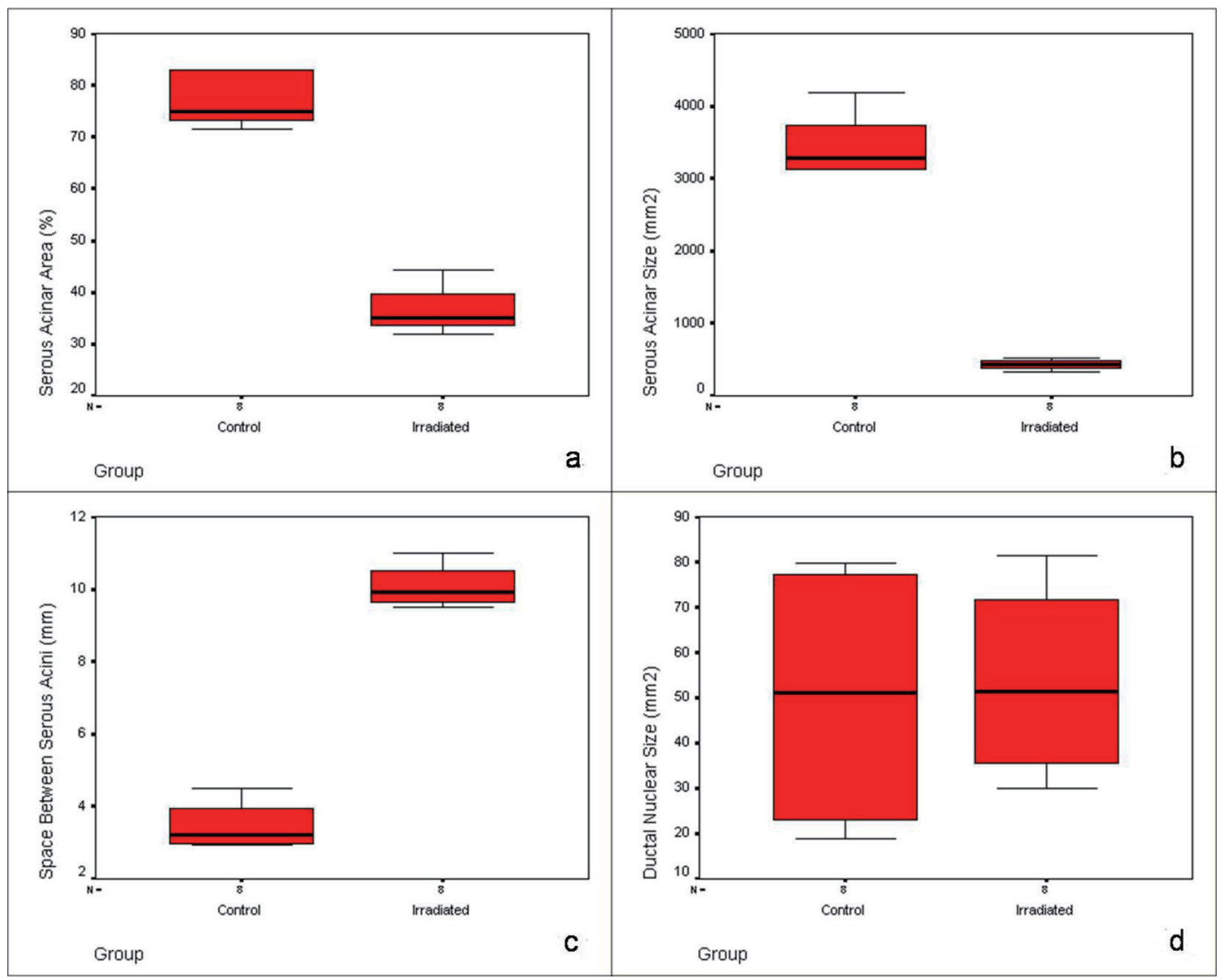

Figure 4. Box plots showing the pattern and interquartile range of histomorphometric parameters in control and irradiated group: (a) serous acinar area, (b) serous acinar size, (c) space between serous acini and (d) ductal nuclear size. 
taining secretory granules that increase the sensitivity of the salivary glands to ionizing radiations and later to its lysis [15]. We also noticed a marked increase in the inter-acinar space mainly due to the acinar shrinkage and accumulation of interacinar edema fluid with no connective tissue sclerosis. This impaired the secretion and explained the sharp drop in saliva in the first 3 - 4 days of radiotherapy $[15,17]$. Histologically, rat submandibular gland resembles humans, except for the GCTs. The granules contain epidermal/neural growth factors and hormones which are exocytosed against neural and hormonal stimuli [16]. Ductal degeneration was a consistent finding in all the irradiated samples, though their nuclei did not appear to be affected. Broadly, radiation effects on glandular tissues are cited to cause hypocellularity, hypovascularity, and hypoxia [18]. For salivary glands, cell death consequent to radiation is ascribed to several factors: apoptosis, necrosis, autophagy, senescence, activation of calcium channels in the cell membrane and loss of stem/progenitor cells, all mediated by regulatory proteins, cytokines, chemokines and several other signaling molecules [19]. All these produce a direct cytotoxic action followed by effects due to acinar cell degranulation and ductal obstruction [13]. Since many of these would set in the later stages, rapid drop in saliva secretion in the early stages of radiotherapy has remained intriguing [20]. Our findings, quantified by morphometry, gave accurate estimates of acinar cell dysfunctions.

Cytoplasmic vacuolization is due to the effect of irradiation on the acinar cell membrane transport mechanisms: early effects are due to cell membrane damages, which cause irregularities in the acinar cells with reduced secretion of amylase [21]. These include disturbances in cell membrane-receptor interactions with muscarinic acetyl choline receptors (M3) and Gq-proteins that increase intracellular calcium levels and thereby the activation of several signal transduction pathways [22]. Aquaporin expression is another pathway related to acinar aberrations. Aquaporin-5 (AQP5) is a water channel present in the acinar cell apical membrane, whose downregulation is reported after irradiation [23]. In our study, round to ovoid shaped vacuoles were seen widely distributed in the irradiated gland parenchyma. This agreed to the previous salivary gland investigations [16, 17, 21].

At higher doses of radiations, DNA damages occur in the salivary glands. In the present study, we observed shrunken nuclei with condensed and clumping chromatins but no nuclear disintegration, which presumably may be due to the dose of 15 Gy that was not sufficiently high enough to cause complete DNA destruction. This showed that cell death due to DNA damages might be appearing after the initial stages of fractionated radiotherapy. Ionizing radiations elicit DNA damage directly through double stand breakages or indirectly through reactive oxygen species (ROS) causing mutations and cell death. Even if the DNA damages do not precipitate in sudden cell death, improper DNA repair can affect acinar cell functions and its future proliferation and regeneration [24]. Since salivary glands are abundant with water, radiation causes its ionization into free radicals like hydroxyl ions and reactive oxygen species such as peroxyl radicals, superoxide anions, and hydrogen peroxide, all of which would cause cell destruction [25]. Cell death mediated through apoptosis is another late event occurring after irradiation by the transcriptional activa- tion of p53, a tumor suppressor gene [26]. Irreversible destructive changes like acinar apoptosis and connective tissue sclerosis develop and continue in the salivary glands throughout the lifetime after irradiation [27]. From our findings, it implied that the sudden drop in salivary output that occurs during the initial few days after a "single animal dose" equaling to "first week of fractionated clinical radiation doses" is predominantly due to acinar malfunctions and to a lesser extent by cell death. Further, understanding the number of undamaged acinar cells immediately after radiotherapy is crucial, because that would determine the regenerative abilities of the gland after irradiation. Several new modes of therapeutic measures are being developed for xerostomia: palliative, protective and regenerative [10]. However, no effective interventions have been developed so far, because of the lack of clarity in preliminary post-radiation histology. Acute radiation effects notably influence the regenerative capacities of stem and progenitor cells [6]. Therefore, clear understanding of the number of cells affected immediately after radiation is important. This is because the diverse type of late effects springs from acute effects [28].

\section{Conclusions}

The morphometry study gave objective estimates in assessing the early radiation-induced changes of the submandibular salivary glands: reduction of acinar area and acinar size. Submandibular salivary gland parenchyma showed discrete differences in radiosensitivity; serous cells were affected but mucus cells were unaffected. The increased inter-acinar space was due to the accumulation of edematous fluid and not with obliteration of connective tissue sclerosis. Though ductal degeneration was noticed, their nuclei did not show major degenerative changes. All these suggested gross acinar cell malfunctions in the submandibular salivary gland during the early irradiation phase. Considering the leading role of submandibulars in maintaining optimum salivation, current findings can form the basis of a comprehensive animal model for: 1) future histologic elements to be focused and protected in IMRT and 2) targeted by novel radioprotectors for reversing effects of radiation-induced xerostomia.

\section{Conflicts of Interest}

None.

\section{References}

1. Gomez DR, Zhung JE, Gomez J, Chan K, Wu AJ, Wolden $\mathrm{SL}$, Pfister DG, et al. Intensity-modulated radiotherapy in postoperative treatment of oral cavity cancers. Int $\mathrm{J} \mathrm{Ra}$ diat Oncol Biol Phys. 2009;73(4):1096-1103.

2. Clark CH, Miles EA, Urbano MT, Bhide SA, Bidmead AM, Harrington KJ, Nutting CM. Pre-trial quality assurance processes for an intensity-modulated radiation therapy (IMRT) trial: PARSPORT, a UK multicentre Phase III trial comparing conventional radiotherapy and parotid- 
sparing IMRT for locally advanced head and neck cancer. Br J Radiol. 2009;82(979):585-594.

3. Grundmann O, Mitchell GC, Limesand KH. Sensitivity of salivary glands to radiation: from animal models to therapies. J Dent Res. 2009;88(10):894-903.

4. Dirix P, Nuyts S, Vander Poorten V, Delaere P, Van den Bogaert $\mathrm{W}$. The influence of xerostomia after radiotherapy on quality of life: results of a questionnaire in head and neck cancer. Support Care Cancer. 2008;16(2):171-179.

5. Vissink A, van Luijk P, Langendijk JA, Coppes RP. Current ideas to reduce or salvage radiation damage to salivary glands. Oral Dis. 2015;21(1):e1-10.

6. de Almeida Pdel V, Gregio AM, Machado MA, de Lima AA, Azevedo LR. Saliva composition and functions: a comprehensive review. J Contemp Dent Pract. 2008;9(3):72-80.

7. Li Y, Taylor JM, Ten Haken RK, Eisbruch A. The impact of dose on parotid salivary recovery in head and neck cancer patients treated with radiation therapy. Int J Radiat Oncol Biol Phys. 2007;67(3):660-669.

8. Globus RK, Caiozzo VJ, Acharya MM, Fike JR, Limoli CL. Redox regulation of stem cell compartments: the convergence of radiation induced normal tissue damage and oxidative stress. In: Spitz DR, Dornfeld, KJ, Gius D, Krishnan K. Oxidative Stress in Applied Basic Research and Clinical Practice: Cancer. Totowa, NJ: Humana Press, 2012; p. 169-192.

9. Jha N, Harris J, Seikaly H, Jacobs JR, McEwan AJ, Robbins KT, Grecula J, et al. A phase II study of submandibular gland transfer prior to radiation for prevention of radiation-induced xerostomia in head-and-neck cancer (RTOG 0244). Int J Radiat Oncol Biol Phys. 2012;84(2):437-442.

10. Rosen EM, Day R, Singh VK. New approaches to radiation protection. Front Oncol. 2014;4:381.

11. Hosseinimehr SJ. Trends in the development of radioprotective agents. Drug Discov Today. 2007;12(19-20):794805.

12. Murdoch-Kinch CA, Kim HM, Vineberg KA, Ship JA, Eisbruch A. Dose-effect relationships for the submandibular salivary glands and implications for their sparing by intensity modulated radiotherapy. Int J Radiat Oncol Biol Phys. 2008;72(2):373-382.

13. El-Mofty SK, Kahn AJ. Early membrane injury in lethally irradiated salivary gland cells. Int J Radiat Biol Relat Stud Phys Chem Med. 1981;39(1):55-62.

14. Abok K, Brunk U, Jung B, Ericsson J. Morphologic and histochemical studies on the differing radiosensitivity of ductular and acinar cells of the rat submandibular gland. Virchows Arch B Cell Pathol Incl Mol Pathol.
1984;45(4):443-460.

15. Nagler RM, Baum BJ, Miller G, Fox PC. Long-term salivary effects of single-dose head and neck irradiation in the rat. Arch Oral Biol. 1998;43(4):297-303.

16. El-Direny EA, Shalaby NM, Afif OK. Effect of irradiation on secretory cells of submandibular gland of adult male albino rat. Histological and immunohistochemical study. Egypt J Histol. 2009;32(2):324-332.

17. Burlage FR, Coppes RP, Meertens H, Stokman MA, Vissink A. Parotid and submandibular/sublingual salivary flow during high dose radiotherapy. Radiother Oncol. 2001;61(3):271-274.

18. Marx RE. Osteoradionecrosis: a new concept of its pathophysiology. J Oral Maxillofac Surg. 1983;41(5):283-288.

19. Konings AW, Faber H, Cotteleer F, Vissink A, Coppes RP. Secondary radiation damage as the main cause for unexpected volume effects: a histopathologic study of the parotid gland. Int J Radiat Oncol Biol Phys. 2006;64(1):98105.

20. Nagler RM. The enigmatic mechanism of irradiationinduced damage to the major salivary glands. Oral Dis. 2002;8(3):141-146.

21. Zeilstra LJ, Vissink A, Konings AW, Coppes RP. Radiation induced cell loss in rat submandibular gland and its relation to gland function. Int J Radiat Biol. 2000;76(3):419429.

22. Coppes RP, Zeilstra LJ, Kampinga HH, Konings AW. Early to late sparing of radiation damage to the parotid gland by adrenergic and muscarinic receptor agonists. $\mathrm{Br}$ J Cancer. 2001;85(7):1055-1063.

23. Li Z, Zhao D, Gong B, Xu Y, Sun H, Yang B, Zhao X. Decreased saliva secretion and down-regulation of AQP5 in submandibular gland in irradiated rats. Radiat Res. 2006;165(6):678-687.

24. Slupphaug G, Kavli B, Krokan HE. The interacting pathways for prevention and repair of oxidative DNA damage. Mutat Res. 2003;531(1-2):231-251.

25. Wardman P, Ross AB. "Radiation chemistry" literature compilations: their wider value in free radical research. Free Radic Biol Med. 1991;10(3-4):243-247.

26. Limesand KH, Said S, Anderson SM. Suppression of radiation-induced salivary gland dysfunction by IGF-1. PLoS One. 2009;4(3):e4663.

27. De la Cal C, Fernandez-Solari J, Mohn C, Prestifilippo J, Pugnaloni A, Medina V, Elverdin J. Radiation produces irreversible chronic dysfunction in the submandibular glands of the rat. Open Dent J. 2012;6:8-13.

28. O'Connell AC. Natural history and prevention of radiation injury. Adv Dent Res. 2000;14:57-61. 